\title{
ON THE ZEROS OF SOLUTIONS OF LINEAR DIFFERENTIAL EQUATIONS OF THE SECOND ORDER
}

\author{
WALTER BERGWEILER AND NORBERT TERGLANE
}

\begin{abstract}
Let $u$ be a solution of the differential equation $u^{\prime \prime}+R u=0$, where $R$ is rational. Newton's method of finding the zeros of $u$ consists of iterating the function $f(z)=z-u(z) / u^{\prime}(z)$. With suitable hypotheses on $R$ and $u$, it is shown that the iterates of $f$ converge on an open dense subset of the plane if they converge for the zeros of $R$. The proof is based on the iteration theory of meromorphic functions, and in particular on the result that, if the family of $K$-quasiconformal deformations of a meromorphic function $f$ depends on only finitely many parameters, then every cycle of Baker domains of $f$ contains a singularity of $f^{-1}$. This result, together with classical results of Hille concerning the asymptotic behaviour of solutions of the above differential equations, is also used to study their value distribution. For example, it is shown that, if $R$ is a rational function which satisfies $R(z) \sim a_{m} z^{m}$ as $z \rightarrow \infty$ and has only $k$ distinct zeros where $k<(m+2) / 2$, then $\delta(0, u) \leqslant k /(m+2-k)<1$.
\end{abstract}

\section{Newton's method}

We consider the differential equation

$$
w^{\prime \prime}+p w^{\prime}+q w=0
$$

where the coefficients $p$ and $q$ are rational functions. The well known substitution

$$
u=w \exp \left(\frac{1}{2} \int^{z} p(t) d t\right)
$$

leads to the differential equation

$$
u^{\prime \prime}+R u=0
$$

where

$$
R=q-\frac{1}{4} p^{2}-\frac{1}{2} p^{\prime}
$$

We shall assume that $R \not \equiv 0$ so that $u$ is not linear or constant. If $R$ has a multiple pole $z_{0}$, then $v(z)=z u\left(z_{0}+1 / z\right)$ satisfies $v^{\prime \prime}+S v=0$, where $S(z)=R\left(z_{0}+1 / z\right) / z^{4} \neq o\left(1 / z^{2}\right)$ as $z \rightarrow \infty$. Thus it is no loss of generality to restrict to the case in which $R$ has only simple poles or satisfies

$$
R(z) \neq o\left(\frac{1}{z^{2}}\right)
$$

as $z \rightarrow \infty$. We note that (4) is satisfied if (3) has a transcendental meromorphic solution (see, for example, [33, $\S 5 ; \mathbf{4 0}, \S 3]$ ). (Here and in the following, 'meromorphic' will always mean meromorphic in the plane, unless a domain is specified.)

An important problem is to study the zeros of solutions of (1) and (3). Here we say that $z_{0}$ is a zero of $u$ if some branch of $u$ is analytic in a neighbourhood of $z_{0}$ and $u\left(z_{0}\right)=0$ for this branch. We note that, if $u$ and $w$ are related by (2), then any zero

Received 31 August 1995.

1991 Mathematics Subject Classification 30D05.

The first author was supported by a Heisenberg Fellowship of the Deutsche Forschungsgemeinschaft.

J. London Math. Soc. (2) 58 (1998) 311-330 
$z_{0}$ of $u$ is also a zero of $w$ and vice versa, except possibly if $z_{0}$ is a pole of $p$. Therefore it suffices to consider differential equations of form (3). We also note that, even if $w$ is a meromorphic function, $u$ need not be meromorphic. However, we have

$$
\frac{u^{\prime}}{u}=\frac{w^{\prime}}{w}+\frac{1}{2} p
$$

and thus $u / u^{\prime}$ is meromorphic if and only if $w / w^{\prime}$ is. We shall assume throughout that this is the case.

Newton's method of finding the zeros of $u$ consists of iterating the function

$$
f(z)=z-\frac{u(z)}{u^{\prime}(z)} .
$$

In terms of $w$, we have

$$
f(z)=z-\frac{2 w(z)}{2 w^{\prime}(z)+p(z) w(z)} .
$$

Denote by $f^{n}$ the $n$th iterate of $f$. If $z_{0}$ is a zero of $u$, then $f^{n}(z) \rightarrow z_{0}$ as $n \rightarrow \infty$ for all $z$ sufficiently close to $z_{0}$. If, in addition, $z_{0}$ is not a pole of $R$, then $z_{0}$ is a simple zero of $u$ because otherwise the uniqueness of solutions yields $u \equiv 0$. It follows that $z_{0}$ is a multiple zero of $f^{\prime}=u^{\prime \prime} u /\left(u^{\prime}\right)^{2}=-R\left(u / u^{\prime}\right)^{2}$ so that $f(z)=z_{0}+O\left(\left(z-z_{0}\right)^{3}\right)$ as $z \rightarrow z_{0}$ in this case. This implies that the convergence to $z_{0}$ is of the third order. We note that, in general, Newton's method converges only quadratically to simple zeros; that is, for solutions of (3), the local behaviour of Newton's method near zeros is better than for general meromorphic functions.

In this paper, however, we are mainly interested in the global aspects of Newton's method applied to solutions of (3). From this point of view, it is of interest that there are simple examples (see, for example, $[\mathbf{1 1}, \S 2]$ and Examples 5 and 6 in $\S 9$ of this paper) where Newton's method fails to converge on some open set.

THEOREM 1. Let $R$ be a rational function having only simple poles or satisfying (4), and denote by $z_{1}, \ldots, z_{N}$ the finite zeros of $R$. Let $u$ be a solution of (3) such that $u / u^{\prime}$ is meromorphic, and define $f$ by (5). Suppose that $f^{n}\left(z_{j}\right)$ converges to a finite limit as $n \rightarrow \infty$ for all $j \in\{1,2, \ldots, N\}$. If $u / u^{\prime}$ is transcendental, then $f^{n}(z)$ converges to zeros of $u$ or finite poles of $R$ on an open dense subset of $\mathbb{C}$. If $u$ is meromorphic but not of the form $u(z)=\exp (a z+b)$ for some $a, b \in \mathbb{C}$, then $f^{n}(z)$ converges to zeros of $u$ on an open dense subset of $\mathbb{C}$.

If $u / u^{\prime}$ is rational and $u$ is not meromorphic, then not only does the case in which $u(z)=\exp (a z+b)$ have to be excluded, but there are further exceptional cases (see the remarks at the end of $\S 6$ and Example 4 in $\S 9$ ).

In the case in which $z_{0}$ is a pole of $R$, one can easily determine from the Laurent series of $R$ and $u / u^{\prime}$ whether there is an open set where $f^{n}$ converges to $z_{0}$ (see Lemma 2 in $\S 8)$.

This paper is organised as follows. In $\S 2$ we study the asymptotic behaviour and the value distribution of meromorphic solutions $u$ of (3). In particular, we give some upper bounds for the Nevanlinna deficiency of the zeros of $u$. In $\S 3$ we state some results from iteration theory on which the proofs of Theorem 1 and the results of $\S 2$ are based. These results include conditions implying that cycles of Baker domains contain a singularity of the inverse function. They are proved in $\$ 4$ and $\S 5$, and 
Theorem 1 is then proved in $\S 6$. The results of $\S 2$ are proved in $\S 7$. In $\S 8$ we consider the behaviour of $u$ and $f$ near poles of $R$. Finally, in $\$ 9$, we consider some examples, for example Bessel functions.

\section{Value distribution theory}

The methods used in the proof of Theorem 1 also give information on the frequency of the zeros of solutions of (3). More specifically, we shall obtain upper bounds for the Nevanlinna deficiency $\delta(0, u)$ if $u$ is meromorphic and satisfies (3). For the notations and basic results of Nevanlinna theory, we refer to $[\mathbf{2 4}, \mathbf{3 1}, \mathbf{3 3}, \mathbf{3 6}$.

Assume that

$$
R(z) \sim a_{m} z^{m}
$$

as $z \rightarrow \infty$ where $m$ is an integer and $a_{m} \neq 0$. Further suppose that $u$ is a transcendental meromorphic solution of (3). Then $m \geqslant-1$ so that (4) is satisfied and the order $\rho(u)$ of $u$ is given by

$$
\rho=\frac{m+2}{2}
$$

(see, for example, $[\mathbf{3 3}, \S 5 ; \mathbf{4 0}, \S 2])$. Clearly, $u$ has at most finitely many poles.

We shall use some results of Hille $[\mathbf{2 6}, \mathbf{2 7}$ ] (see also $[35,40]$ ) on the asymptotic behaviour of solutions of (3). In order to describe these results, we assume that $a_{m}=-((m+2) / 2)^{2}$ so that

$$
R(z) \sim a_{m} z^{m}=-\left(\frac{m+2}{2}\right)^{2} z^{m}=-\rho^{2} z^{m}
$$

as $z \rightarrow \infty$. This is no loss of generality, as it can be achieved by considering $u(c z)$ instead of $u(z)$ where $c^{m+2}=-\rho^{2} / a_{m}$. For fixed $\varepsilon$ satisfying $0<\varepsilon<\pi /(m+2)$ and $j \in\{0,1, \ldots, m+1\}$, we consider the sectors

$$
S_{j}=\left\{z:\left|\arg z-\frac{2 \pi j}{m+2}\right|<\frac{\pi}{m+2}-\varepsilon\right\} .
$$

It is a consequence of Hille's basic result that for each $j$ there exists $\varepsilon_{j} \in\{ \pm 1\}$ such that

$$
\log u(z) \sim \varepsilon_{j} z^{(m+2) / 2}=\varepsilon_{j} z^{\rho}
$$

uniformly as $z \rightarrow \infty$ in $S_{j}$. In particular, either $|u(z)| \rightarrow 0$ in $S_{j}$ or $|u(z)| \rightarrow \infty$ in $S_{j}$. If $|u(z)| \rightarrow 0$ in $S_{j}$, then $|u(z)| \rightarrow \infty$ in $S_{j-1}$ and in $S_{j+1}$. Here we use the convention that $S_{-1}=S_{m+1}$ and $S_{m+2}=S_{0}$. Denote by $p$ the number of sectors where $|u(z)| \rightarrow 0$, and denote by $q$ the number of sectors where $|u(z)| \rightarrow \infty$. Then $p+q=m+2$ and $p \leqslant q$. For the quantities of Nevanlinna theory, we obtain

$$
T(r, u) \sim m(r, u) \sim \frac{q}{\pi \rho} r^{\rho}
$$

and

as $r \rightarrow \infty$. This implies that

$$
m\left(r, \frac{1}{u}\right) \sim \frac{p}{\pi \rho} r^{\rho}
$$

$$
\delta(0, u)=\frac{p}{q}=\frac{p}{m+2-p}=\frac{p}{2 \rho-p} .
$$

In particular, $\delta(0, u)$ is always of form (9) for some integer $p$ satisfying $0 \leqslant p \leqslant \rho$. We may, of course, take (9) as the definition of $p$ if $\delta(0, u)$ and $m$ are given. 
THEOREM 2. Let u be a meromorphic solution of (3) with $R$ satisfying (8). Suppose that $u(z) \neq \exp (a z+b)$ with $a, b \in \mathbb{C}$. Define $f$ by (5) and $p$ by (9). Then there exist zeros $z_{1}, \ldots, z_{p}$ of $R$ such that

$$
\lim _{n \rightarrow \infty} \frac{\left|f^{n}\left(z_{k}\right)\right|^{\rho}}{n}=1
$$

for $k=1, \ldots, p$, where $\rho=(m+2) / 2$ is the order of $u$. In particular,

$$
\lim _{n \rightarrow \infty}\left|f^{n}\left(z_{k}\right)\right|=\infty
$$

for $k=1, \ldots, p$.

COROLlary 1. Let $u$ be a meromorphic solution of (3) for some rational $R$. Suppose that the hypotheses of Theorem 1 are satisfied, that is, if $R\left(z_{0}\right)=0$, then $f^{n}\left(z_{0}\right)$ converges to a finite limit. Then $\delta(0, u)=0$.

COROllary 2. Let $u$ be a meromorphic solution of (3) for some rational $R$ satisfying (7). Denote by $k$ the number of distinct zeros of $R$, and suppose that $k<$ $(m+2) / 2=\rho$. Then $\delta(0, u) \leqslant k /(m+2-k)<1$.

For example, we see that, if

$$
u^{\prime \prime}(z)-z^{m} u(z)=0,
$$

then $\delta(0, u) \leqslant 1 /(m+1)$. This follows also from the result in [23]. In [6, $\S 4]$ it was shown that solutions of (11) cannot have 0 as a Borel exceptional value.

\section{Iteration theory}

Let $f$ be a rational or transcendental meromorphic function. The Fatou set $F(f)$ is the set of all $z \in \hat{\mathbb{C}}$ which have a neighbourhood $U$ such that all iterates $f^{n}$ are meromorphic in $U$ and form a normal family there. The complement of the Fatou set is called the Julia set of $f$. For an introduction to iteration theory, we refer to the books $[7, \mathbf{1 4}, 38]$ and the lecture notes [34] for rational functions, and to the survey article [9] for transcendental functions. The classical papers are [19, 20, 32].

Let $U$ be a component of $F(f)$. Then $f^{n}(U)$ is contained in some component $U_{n}$ of $F(f)$, with the convention that $U_{0}=U$. One says that $U$ is a wandering domain if $U_{m} \neq U_{n}$ for all $m \neq n$. Otherwise, $U$ is called preperiodic. If $U=U_{l}$ for some $l \in \mathbb{N}$, then $U$ is called periodic, and the smallest $l$ satisfying $U=U_{l}$ is called the period of $U$.

One of the basic results in iteration theory is Theorem A, which is due to Sullivan [39].

THEOREM A. Rational functions do not have wandering domains.

This result has been generalised to various classes of transcendental entire and meromorphic functions (see $[\mathbf{2}, \mathbf{5}, \mathbf{8}, \mathbf{1 1}, \mathbf{1 2}, \mathbf{1 6}, \mathbf{1 8}, \mathbf{2 2}, \mathbf{3 7}]$ ). For our purposes, the following generalisation of Theorem $\mathrm{A}$ is important [13, Theorem 3].

THEOREM B. Let $f$ be a meromorphic function which satisfies the Riccati equation

$$
f^{\prime}(z)+R(z)(f(z)-z)^{2}=0
$$

where $R$ is rational. Then $f$ does not have wandering domains. 
Note that, if $u$ satisfies (3) and if $f$ is defined by (5), then (12) holds.

For functions which do not have wandering domains, it suffices to consider periodic components of the Fatou set. The limiting behaviour of the iterates in periodic components is well understood. For rational functions, a periodic component of the Fatou set is a (super)attracting component, a parabolic component, a Siegel disc, or a Herman ring. We refer to $[\mathbf{7}, \S 7.1]$ for the terminology used and a proof of this classification of periodic components. The result can also be found in [14, §IV.2] and $[38, \S 3.2]$, where a slightly different terminology is used.

For transcendental functions, the only further possibility for a periodic component of the Fatou set is that of a Baker domain, also called an essentially parabolic domain (see [3, Theorems 2.2 and 2.3; 9, Theorem 6]). By definition, a periodic component $U$ of $F(f)$ of period $l$ is called a Baker domain if $\left.f^{l n}\right|_{U} \rightarrow z_{0} \in \partial U$ as $n \rightarrow \infty$, and $f^{l}\left(z_{0}\right)$ is not defined. For $l=1$ or for entire $f$, this is possible only if $z_{0}=\infty$, but, in general, $z_{0}$ can also be a pole of $f^{k}$ for some $k \leqslant l-1$.

The periodic components that occur in the iteration of rational functions are closely related to the singularities of the inverse function $f^{-1}$ of $f$, that is, to the critical and asymptotic values of $f$ (see, for example, $[9, \S 4.3]$ ). On the other hand, there do not seem to be close relations between Baker domains and critical or asymptotic values (compare $[\mathbf{1 0}, \mathbf{1 7}, \mathbf{2 5}])$. However, we have the following result.

THEOREM 3. Let $f$ be a transcendental meromorphic function satisfying (12) with some rational function $R$. If $U$ is a Baker domain of period l of $f$, then $\bigcup_{j=0}^{l-1} U_{j}$ contains a critical or asymptotic value of $f$.

Sullivan's proof [39] of Theorem A uses the theory of $K$-quasiconformal mappings. Let $f$ be meromorphic and $\Phi$ be a $K$-quasiconformal mapping of the sphere, and suppose that $f_{\Phi}=\Phi \circ f \circ \Phi^{-1}$ is also meromorphic. Then $f_{\Phi}$ is called a $K$ quasiconformal deformation of $f$. If $f$ is rational, then so is $f_{\Phi}$ [39, Proposition 8]. In fact, $f$ and $f_{\Phi}$ have the same degree then, and thus the family of $K$-quasiconformal deformations of $f$ depends (real analytically) on only finitely many (real) parameters. This fact plays a key role in Sullivan's proof of Theorem A. Most results concerning the non-existence of wandering domains for transcendental functions exploit the fact that, for certain classes of functions, the family of $K$-quasiconformal deformations depends on only finitely many parameters. (An exception is [11].) In particular, it was shown in $[11, \S 4.3]$ that this is the case for functions satisfying the hypotheses of Theorem 3. Thus Theorem 3 is a consequence of the following result.

THEOREM 4. Let $f$ be a transcendental meromorphic function, and suppose that the family of $K$-quasiconformal deformations of $f$ depends on only finitely many parameters. Then the conclusion of Theorem 3 holds.

We note that Herman [25, §III.9] has stated this result (without proof) for entire functions. The case of entire functions is somewhat easier, because here preperiodic components of the Fatou set, and thus in particular Baker domains, are always simply connected by a result of Baker [2, Theorem 3.1].

In [8, Theorem 2; 9, Theorem 14], it was shown that the conclusion of Theorem 3 holds for certain other classes of meromorphic function. The results given there also follow from Theorem 4. 


\section{Proof of Theorem 4}

Our proof is based on the ideas introduced by Sullivan [39] in his proof of Theorem A. There, the case of a wandering annulus was considered separately $[39, \S 2]$. Here we have the following result.

LEMMa 1. Let $f$ be a meromorphic function with a doubly connected Baker domain $U$ of period l. Then $\bigcup_{j=0}^{l-1} U_{j}$ contains a critical or asymptotic value of $f$.

Proof. For a suitable $r>0$, there exists a Riemann map $\psi: U \rightarrow A=$ $\{z: r<|z|<1\}$. Define $g=\psi \circ f^{l} \circ \psi^{-1}$. If $\bigcup_{j=0}^{l-1} U_{j}$ does not contain a critical or asymptotic value of $f$, then $f^{l}: U \rightarrow U$, and thus $g: A \rightarrow A$ is an unramified covering. We deduce that $g(z)=\mathrm{e}^{\mathrm{i} \alpha} z$ or $g(z)=\mathrm{e}^{\mathrm{i} \alpha} r / z$ for some $\alpha \in \mathbb{R}$. This implies that the iterates of $\left.g\right|_{A}$ do not tend to the boundary of $A$, and thus there does not exist $z_{0} \in \partial U$ such that $\left.f^{l n}\right|_{U} \rightarrow z_{0}$ as $n \rightarrow \infty$, contradicting the hypothesis that $U$ is a Baker domain. This contradiction completes the proof of Lemma 1 .

We remark that Baker, Kotus, and Lü [4, Theorem 3.1] have shown that invariant Baker domains are either simply or infinitely connected, regardless of whether or not they contain critical or asymptotic values.

In [39, Proposition 3], Sullivan proved Proposition A.

Proposition A. If $S$ is a hyperbolic Riemann surface of infinite topological type, then there are arbitrarily large dimensional families of deformations of the hyperbolic structures so that each pair of surfaces in the family is quasiconformally (even quasiisometrically) isomorphic.

Proposition B has been given by Sullivan [39, Proposition 4] under the assumption that the rational function $f$ has a wandering domain $U$. However, a look at the proofs reveals the following.

Proposition B. Let $f$ be a transcendental meromorphic function, and let $U$ be a component of $F(f)$. Suppose that the $U_{i}$ are not doubly connected and do not contain critical or asymptotic values of $f$, for all $i \geqslant 0$. Then

(1) either, from some $n$ on, $U_{n+i}$ has finite topological type and for each $i \geqslant 0$ the mapping $f: U_{n+i} \rightarrow U_{n+i+1}$ is a conformal bijection;

(2) or the direct limit $U_{\infty}$ of $f: U_{i} \rightarrow U_{i+1}, i \geqslant 0$, exists and has infinite topological type.

Proof of Theorem 4. Let $\left\{U_{0}, \ldots, U_{l-1}\right\}$ be a cycle of Baker domains of period $l$, and assume that $\bigcup_{j=0}^{l-1} U_{j}$ does not contain a critical or asymptotic value of $f$.

At first let $\left\{U_{0}, \ldots, U_{l-1}\right\}$ satisfy case (1) of Proposition B. Following Sullivan $[39, \S 9]$, we can rule out the possibility of $U_{i}$ being simply connected, since Sullivan shows that, for such a $U_{i}$ to be a wandering domain, this implies that, for each $K>1$, the family of $K$-quasiconformal deformations cannot depend on only finitely many parameters. His proof has been simplified by Baker $[2]$ (see also $[\mathbf{5} ; \mathbf{7}, \S 8 ; \mathbf{1 4}$, $\S I V .1 ; 38, \S 3.1]$ ). It was shown in [8, Theorem 2] (see also [9, Theorem 14] and the remarks following it) how the argument can be modified to yield under our assumption the same conclusion that the family of $K$-quasiconformal deformations of 
$f$ cannot depend on only finitely many parameters. Thus we obtain a contradiction to the hypothesis.

In [39, §10], Sullivan shows how to modify the argument for multiply connected $U_{i}$. In our case, the situation is much simpler, because the case of doubly connected domains is ruled out by Lemma 1, and the number of conformal automorphisms of a multiply connected domain of finite connectivity greater than 2 is finite (see, for example, [30, p. 583]). Thus, if the connectivity is greater than 2 , then the sequence $\left(\left.f^{n l}\right|_{U}\right)$ is eventually periodic, say $\left.f^{m l}\right|_{U}=\left.f^{n l}\right|_{U}$ where $m>n$. It follows that $\left.f^{(m-n) l}\right|_{U_{n}}=\left.\mathrm{id}\right|_{U_{n}}$, which is clearly impossible for transcendental meromorphic $f$.

In $[39, \S 10]$, Sullivan also deals with case (2) of Proposition B. Applying Proposition A to $S=U_{\infty}$, he shows that the family of $K$-quasiconformal deformations of $f$ cannot depend on only finitely many parameters. The argument given there extends to our case without modification. Again we find that the family of $K$ quasiconformal deformations of $f$ cannot depend on only finitely many parameters, contradicting the hypothesis.

Hence the proof of Theorem 4 is complete.

\section{Proof of Theorem 3}

In view of Theorem 4, we only have to prove that the family of $K$-quasiconformal deformations of $f$ depends on only finitely many parameters. (This has been proved in $[11, \S 4.3]$, but for the convenience of the reader we give a short outline of the proof here.)

Let $f$ be a transcendental meromorphic function and let $\Phi$ be a $K$-quasiconformal self-map of the Riemann sphere such that $f_{\Phi}=\Phi \circ f \circ \Phi^{-1}$ is meromorphic in $\mathbb{C}$. Because of the Riccati equation (12), all zeros of $f^{\prime}$ are double and are fixpoints of $f$ and vice versa, with the exception of at most finitely many. The same is then true with $f$ replaced by $f_{\Phi}$, and hence $f_{\Phi}^{\prime}(z) /\left(f_{\Phi}(z)-z\right)^{2}$ has only finitely many zeros and poles. Since $f$ is of finite order $\rho(f)$ (see [31, Satz 22.4]), and since $\Phi$ is Hölder continuous with exponent $1 / K$, we find that $f_{\Phi}$, and hence $f_{\Phi}^{\prime}(z) /\left(f_{\Phi}(z)-z\right)^{2}$, is of order at most $K \rho(f)$.

This yields $f_{\Phi}^{\prime}(z) /\left(f_{\Phi}(z)-z\right)^{2}=R_{\Phi}(z) \exp \left(P_{\Phi}(z)\right)$ for some rational $R_{\Phi}$ of the same degree as $R$ and some polynomial $P_{\Phi}$ of degree less than or equal to $K \rho(f)$. We conclude that the family of $K$-quasiconformal deformations of $f$ depends on only finitely many parameters, and therefore the proof is complete.

\section{Proof of Theorem 1}

Let $u$ be a solution of (3) such that $u / u^{\prime}$ is meromorphic, and let $f$ be defined by (5). Then $f$ satisfies the Riccati equation (12). We shall use some results from iteration theory which do not hold for Möbius transformations and constant functions. Therefore we consider the case in which $f$ is a Möbius transformation or a constant function first.

If $f$ is constant, then $R \equiv 0$ by (12), contradicting the hypothesis. If $f(z)=$ $(\alpha z+\beta) /(\gamma z+\delta)$ where $\alpha \delta-\beta \gamma \neq 0$ and $\gamma \neq 0$, then (12) implies that $R(z)=O\left(1 / z^{4}\right)=$ $o\left(1 / z^{2}\right)$ as $z \rightarrow \infty$. Moreover, $f$ has a finite fixpoint which, by (12), is a multiple pole of $R$. Hence $R$ does not satisfy the hypothesis. Thus $f$ has the form $f(z)=\alpha z+\beta$ where $\alpha \neq 0$. If $\alpha=1$, then $u / u^{\prime} \equiv-\beta$, and thus $u$ has the form $u(z)=\exp (a z+b)$ excluded in the hypothesis. If $\alpha \neq 0,1$, then $u^{\prime}(z) / u(z)=\lambda /(z-\mu)$ where $\lambda=1 /(1-\alpha)$ and $\mu=\beta \lambda$. Since the conclusion concerns (for rational $u / u^{\prime}$ ) only the case in which $u$ is 
meromorphic, we assume that this is the case. Then $\lambda$ is an integer and we obtain $u(z)=c(z-\mu)^{\lambda}$ for some constant $c$. If $\lambda$ is positive, then $\mu$ is a zero of $u$ and $|\alpha|<1$. We find that $f^{n}(z) \rightarrow \mu$ for all $z \in \mathbb{C}$. If $\lambda$ is negative, then $\infty$ is a zero of $u$, and $f^{n}(z) \rightarrow \infty$ for all $z \in \mathbb{C} \backslash\{\mu\}$, since $|\alpha|>1$.

We now consider the case in which $f$ is not constant and not a Möbius transformation. The hypotheses of Theorem $\mathrm{B}$ are fulfilled, and thus $f$ has no wandering domains. Therefore we can restrict our attention to periodic components of $F(f)$. Let $\left\{U_{0}, \ldots, U_{l-1}\right\}$ be a periodic cycle of components of $F(f)$ of period $l$.

We shall use relations between this cycle and the singularities of $f^{-1}$. In [11, §4.3], it has been shown that transcendental meromorphic solutions of the Riccati equation (12) do not have asymptotic values. If $\infty$ is a critical point, then $f$ is rational and (12) implies that $R(z)=o\left(1 / z^{2}\right)$ as $z \rightarrow \infty$. It is classical [19, I, p. 168; 32, pp. 85, 243] (see also [34, Corollary 9.4]) that a rational function of degree at least 2 has a fixpoint which is repelling or has multiplier 1 . Thus, if $\infty$ is a critical point of $f$, then $f$ has a finite fixpoint $\zeta_{0}$ which is repelling or satisfies $f^{\prime}\left(\zeta_{0}\right)=1$. From (12), we deduce that $\zeta_{0}$ is a multiple pole of $R$. Hence we find that $R$ does not satisfy the hypothesis if $\infty$ is a critical point. The finite critical points of $f$ are the zeros of $f^{\prime}=u u^{\prime \prime} /\left(u^{\prime}\right)^{2}=$ $-R\left(u / u^{\prime}\right)^{2}$. Since the zeros of $u / u^{\prime}$ are the fixpoints of $f$, we conclude that only the finite zeros of $R$ can lead to critical points of $f$ which are not attracted by zeros of $u / u^{\prime}$.

It is well known (see, for example, $[9, \S 4.3]$ ), that the closure of the forward orbits of the singularities of $f^{-1}$ contains the boundary of any cycle of Siegel discs or Herman rings From the assumption that $f^{n}\left(z_{j}\right)$ converges as $n \rightarrow \infty$ for all zeros $z_{j}$ of $R$, we thus deduce that $\left\{U_{0}, \ldots, U_{l-1}\right\}$ cannot be a cycle of Siegel discs or Herman rings.

Hence, by the classification of periodic components mentioned in $\$ 3$, there remains the possibility of a (super)attracting cycle, a parabolic cycle, or a cycle of Baker domains. For (super)attracting and parabolic cycles, it is well known that $\bigcup_{j=0}^{l-1} U_{j}$ contains a singularity of $f^{-1}$, and, because of Theorem 3 , this holds in our case also for cycles of Baker domains. Thus there is a critical value of $f$ contained in $\bigcup_{j=0}^{l-1} U_{j}$, and, in fact, $\bigcup_{j=0}^{l-1} U_{j}$ also contains the corresponding critical point.

If $\bigcup_{j=0}^{l-1} U_{j}$ contains a zero of $u / u^{\prime}$, then, as already mentioned, we have $l=1$ and $f^{n}(z)$ converges to this zero for all $z \in U_{0}$. It remains to consider the case in which there exists $k, 1 \leqslant k \leqslant N$, such that $z_{k} \in \bigcup_{j=0}^{l-1} U_{j}$. By hypothesis, $f^{n}\left(z_{k}\right) \rightarrow z_{0}$ as $n \rightarrow \infty$ for some $z_{0} \in \mathbb{C}$. Thus $\left.f^{n}\right|_{U_{j}} \rightarrow z_{0}$ as $n \rightarrow \infty$ for all $j \in\{0, \ldots, l-1\}$. On the other hand, if $U_{0}$ is a Baker domain, then there exist some $j \in\{0, \ldots, l-1\}$ such that $\left.f^{n l}\right|_{U_{j}} \rightarrow \infty$ as $n \rightarrow \infty$. Thus $U_{0}$ cannot be a Baker domain. Moreover, since $\left.f^{n}\right|_{U_{j}} \rightarrow z_{0}$ as $n \rightarrow \infty$ for all $j \in\{0, \ldots, l-1\}$, we see that $z_{0}$ is a fixpoint of $f$. We have either $\left|f^{\prime}\left(z_{0}\right)\right|<1$, in which case $l=1$ and $U_{0}$ is a (super)attracting component, or $f^{\prime}\left(z_{0}\right)^{l}=1$, in which case $\left\{U_{0}, \ldots, U_{l-1}\right\}$ is a cycle of parabolic domains. Clearly, $z_{0}$ is a zero of $u / u^{\prime}$. Since each singularity of $u$ is a pole of $R$, this implies that $z_{0}$ is a zero or pole of $u$ or a pole of $R$. Since a pole of $u$ of multiplicity $k$ is a fixpoint of $f$ of multiplier $1+1 / k$, and hence a repelling fixpoint of $f$, there are no open sets where $f^{n}$ converges to poles of $u$. It follows that $z_{0}$ is a zero of $u$ or a pole of $R$, and we conclude that $f^{n}(z)$ converges to zeros of $u$ or finite poles of $R$ for all $z \in F(f)$. Moreover, if $u$ is meromorphic, then finite poles of $R$ are zeros or poles of $u$, and the above reasoning shows that $f^{n}(z)$ converges to zeros of $u$ for all $z \in F(f)$ in this case.

Because the Fatou set $F(f)$ is either a dense subset of $\hat{\mathbb{C}}$ or empty, we have to show that $F(f) \neq \varnothing$. First suppose that $v=u / u^{\prime}$ is transcendental. From (3), we deduce that $v$ satisfies the Riccati equation $v^{\prime}=1+R v^{2}$. This shows that, if $v$ has only finitely many zeros, then $v^{\prime}$ takes the value 1 only finitely often. However, this is impossible 
by [24, Theorem 3.5]. Thus $v$ has infinitely many zeros, and hence $f$ has infinitely many fixpoints. The Riccati equation (12) shows that infinitely many of them are superattracting. Hence $F(f) \neq \varnothing$ follows.

Now suppose that $u$ is a meromorphic solution of (3). It is immediate that $F(f) \neq \varnothing$ if $u$ has zeros. Hence we assume that $u$ has no zeros. A consideration of the multiplicity of the poles in equation (3) shows that $u$ has poles at most at the multiple poles of $R$ and hence at most finitely many. Since $u$ has finite order (see [31, Satz 22.1; 33, $55 ; \mathbf{4 0}, \S 2])$, this implies that there are polynomials $P, Q$ such that $P u=\mathrm{e}^{Q}$. This leads to the rational function

$$
f(z)=z-\frac{P(z)}{Q^{\prime}(z) P(z)-P^{\prime}(z)} .
$$

If the degree of $Q$ is strictly larger than 1 , or if $Q$ has degree 1 and $P$ is non-constant, then $\infty$ is a rationally indifferent fixpoint of $f$. This implies that there exists a critical point of $f$ and hence a zero of $R$ which tends to $\infty$ under iteration of $f$, contradicting the hypothesis. If $Q$ is constant, then $P$ is non-constant and $\infty$ is an attracting but not superattracting fixpoint of $f(z)=z-P(z) / P^{\prime}(z)$, and again we find that there is a zero of $R$ which tends to $\infty$ under iteration of $f$, contradicting the hypothesis. Thus the degree of $Q$ equals 1 and $P$ is constant. Hence $f$ is linear, and we are in the case already considered. This completes the proof.

The above proof shows that, under the hypotheses of Theorem 1, we have convergence of $f^{n}$ to zeros of $u$ or finite poles of $R$ on an open dense subset of $\mathbb{C}$ as soon as $F(f) \neq \varnothing$, and we have shown that this is always the case if $u / u^{\prime}$ is transcendental. Of course, $F(f) \neq \varnothing$ is also satisfied for many rational functions $u / u^{\prime}$, for example if $u$ has a zero, but it can occur for rational functions $u / u^{\prime}$ that $F(f)=\varnothing$ (see Example 4 in $\S 9$ ).

The exceptional case in which $u(z)=\exp (a z+b)$ is of a different nature. Here we have $f(z)=z-1 / a$ so that $f$ is a linear polynomial. The iteration theory of Möbius transformations is fairly simple [7,§1.2], but, as mentioned in the above proof of Theorem 1, it is somewhat exceptional in complex dynamics. If we do not make the assumption that $u$ is meromorphic, then there are more cases where $f$ is a Möbius transformation and $f^{n}$ does not converge to zeros of $u$ or finite poles of $R$. In the above proof of Theorem 1, we also have to consider the case in which $\lambda$ is not an integer, and we find that we also have to exclude the case in which $u$ has the form $u(z)$ $=c(z-\mu)^{\lambda}$ where $c, \mu \in \mathbb{C}, c \neq 0, \lambda \notin \mathbb{Z}$, and $\operatorname{Re} \lambda \leqslant \frac{1}{2}$.

If we do not hypothesise that $R(z) \neq o\left(1 / z^{2}\right)$ or that $R$ has only simple poles, then there are even more exceptional cases. Here we only mention that, if $u(z)=z /(z-1)$, then $R(z)=-2 /\left(z(z-1)^{2}\right)$ and $f(z)=z^{2}$. Clearly $f^{n}(z) \rightarrow \infty$ as $n \rightarrow \infty$ if $|z|>1$, but $\infty$ is neither a zero of $u$ nor a pole of $R$.

\section{Proof of Theorem 2}

As described before the statement of Theorem 2, we have $p$ sectors $S_{j_{1}}, \ldots, S_{j_{p}}$ such that $|u(z)| \rightarrow 0$ as $z \rightarrow \infty$ in $S_{j_{k}}, k=1, \ldots, p$. We shall prove that for each $k$ there exists a zero $z_{k}$ of $R$ satisfying (10). Moreover, we shall show that the $z_{k}$ have the additional property that $f^{n}\left(z_{k}\right) \in S_{j_{k}}$ for all large $n$. This implies that the $z_{k}$ are pairwise disjoint, and the conclusion of Theorem 2 follows. We shall only consider the case in which $j_{k}=0$ because the other cases are analogous. We thus assume that $|u(z)| \rightarrow 0$ in $S_{0}$. It 
follows that $\log u(z) \sim-z^{\rho}$ as $z \rightarrow \infty$ in $S_{0}$. (In the case in which $m$ is odd so that $\rho$ is not an integer, we have used the branch of $z^{\rho}$ which is positive on the positive real axis.) Let $v(z)=\log u(z)+z^{\rho}$. Then $v(z)=o\left(|z|^{\rho}\right)$ as $z \rightarrow \infty$ in $S_{0}$, and hence

$$
\left|v^{\prime}(z)\right|=\frac{1}{2 \pi}\left|\int_{|\zeta-z|=\eta|z|} \frac{v(\zeta)}{(\zeta-z)^{2}} d \zeta\right| \leqslant \frac{1}{\eta|z|} \max _{|\zeta-z|=\eta|z|}|v(\zeta)|=/\left(|z|^{\rho-1}\right)
$$

as $z \rightarrow \infty$ for $|\arg z| \leqslant \pi /(m+2)-\varepsilon-\arcsin \eta$, provided that $\eta<1$. As $\varepsilon$ and $\eta$ may be chosen arbitrarily small, we can in fact assume that $v^{\prime}(z)=o\left(|z|^{\rho-1}\right)$ as $z \rightarrow \infty$ in $S_{0}$ so that

and hence

$$
\frac{u^{\prime}(z)}{u(z)} \sim-\rho \frac{z^{\rho}}{z}
$$

$$
f(z)=z-\frac{u(z)}{u^{\prime}(z)}=z\left(1+(1+o(1)) \frac{1}{\rho z^{\rho}}\right)
$$

as $z \rightarrow \infty$ in $S_{0}$. Standard arguments now yield the existence of a Baker domain. In fact, the change of variable $\zeta=z^{\rho}$ conjugates $f$ to

$$
F(\zeta)=f\left(\zeta^{1 / \rho}\right)^{\rho}=\zeta\left(1+(1+o(1)) \frac{1}{\rho \zeta}\right)^{\rho}=\zeta\left(1+\frac{1}{\zeta}+o\left(\frac{1}{|\zeta|}\right)\right)=\zeta+1+o(1) .
$$

Define $V=\left\{\zeta:|\zeta|>R_{0},|\arg \zeta|<\pi / 2-\rho \varepsilon\right\}$, where $R_{0}>0$. For sufficiently large $R_{0}$, we have $F(V) \subset V$ and $F^{n}(\zeta) \rightarrow \infty$ for $\zeta \in V$ as $n \rightarrow \infty$. Putting $W=\left\{z: z^{\rho} \in V\right\}$, we find that $f(W) \subset W$ and $f^{n}(z) \rightarrow \infty$ for $z \in W$ as $n \rightarrow \infty$. Thus $W$ is contained in an invariant Baker domain $U$ of $f$. By Theorem 3, $U$ contains a singularity of $f^{-1}$. As proved in $[\mathbf{1 1}, \S 4.3]$ and already used in $\S 6, f$ does not have asymptotic values. Thus $U$ contains a critical value. In fact, $U$ also contains the corresponding critical point $z_{0}$, which must be a zero of $R$.

It is not difficult to see that $f^{n}\left(z_{0}\right) \in W \subset S_{0}$ for all large $n$, say for $n \geqslant N$. Define $\zeta_{n}=f^{n}\left(z_{0}\right)^{\rho}$ for $n \geqslant N$. Then $\zeta_{n+1}=F\left(\zeta_{n}\right)$, and we find from (14) that $\left|\zeta_{n}\right| \sim n$ as $n \rightarrow \infty$. It follows that $\left|f^{n}\left(z_{0}\right)\right|^{\rho} \sim n$, and the proof of Theorem 2 is complete.

We remark that, if we do not normalise as in (8), then we obtain

instead of (10).

$$
\lim _{n \rightarrow \infty} \frac{\left|f^{n}\left(z_{k}\right)\right|^{\rho}}{n}=\frac{\rho}{\sqrt{\left|a_{m}\right|}}
$$

Instead of using Theorem 3, we could also modify a result of Hinkkanen [28, Theorem 2] in the above proof of Theorem 2.

\section{The behaviour of $u$ and $f$ near poles of $R$}

It follows from (12) that poles of $R$ are fixpoints of $f$ and thus zeros of $u / u^{\prime}$. Here we consider the behaviour of $u$ and $f$ near poles of $R$ in more detail.

Lemma 2. Let $z_{0}$ be a pole of multiplicity $k$ of the rational function $R$. Let $u$ be a solution of (3) such that $u / u^{\prime}$ is meromorphic, and define $f$ by (5).

(i) If $k=1$, then $z_{0}$ is a zero of $u$ and an attracting fixpoint of $f$ so that $f^{n}$ converges to $z_{0}$ in some neighbourhood of $z_{0}$. 
(ii) If $k=2$, say

$$
R(z)=\frac{c}{\left(z-z_{0}\right)^{2}}+O\left(\frac{1}{z-z_{0}}\right)
$$

as $z \rightarrow z_{0}$, then

$$
\frac{u(z)}{u^{\prime}(z)}=a\left(z-z_{0}\right)+O\left(\left(z-z_{0}\right)^{2}\right)
$$

as $z \rightarrow z_{0}$, where $c a^{2}-a+1=0$. If $|1-a|<1$ (or, equivalently, if $\operatorname{Re}(1 / a)>\frac{1}{2}$ ), then $z_{0}$ is an attracting fixpoint of $f$ so that $f^{n}$ converges to $z_{0}$ in a neighbourhood of $z_{0}$. If $1-a$ is a root of unity, then $z_{0}$ is a rationally indifferent fixpoint of $f$ so that $f^{n}$ converges to $z_{0}$ in the corresponding parabolic domains. In the remaining cases, there does not exist an open set where $f^{n}$ converges to $z_{0}$. Moreover, $z_{0}$ is a zero of $u$ if and only if $1 / a \in \mathbb{N}$.

(iii) Let $k \geqslant 3$. Then $k$ is even and $z_{0}$ is not a zero of $u$, but a rationally indifferent fixpoint of $f$ so that $f^{n}$ converges to $z_{0}$ in the corresponding parabolic domains.

We note that, if $c a^{2}-a+1=0$, then

$$
\frac{1}{a}=\frac{1}{2} \pm \overline{\frac{1}{4}-c}
$$

Thus, in case (ii), the question of whether there is some open set where $f^{n}$ converges to $z_{0}$ depends not only on $R$ but also on $u$ (see Example 3 in $\S 9$ ).

Proof of Lemma 2. Let $h(z)=\left(z-z_{0}\right)^{k} R(z)$ so that $h\left(z_{0}\right) \neq 0, \infty$ and let $l \in \mathbb{Z}$ and $g$ be analytic in some neighbourhood of $z_{0}$ with $g\left(z_{0}\right) \neq 0, \infty$ such that $u(z) / u^{\prime}(z)=$ $\left(z-z_{0}\right)^{l} g(z)$. The equation

leads to

$$
\left(\frac{u(z)}{u^{\prime}(z)}\right)^{\prime}=1+R(z)\left(\frac{u(z)}{u^{\prime}(z)}\right)^{2}
$$

$$
\left(z-z_{0}\right)^{l-1}\left(\lg (z)+\left(z-z_{0}\right) g^{\prime}(z)-\left(z-z_{0}\right)^{l-k+1} g(z)^{2} h(z)\right) \equiv 1 .
$$

Case (i): Let $k=1$. It follows from (15) that $l=1$ and $g\left(z_{0}\right)=1$ so that $u^{\prime}(z) / u(z)$ $=1 /\left(z-z_{0}\right)+O(1)$. This implies that $z_{0}$ is a (simple) zero of $u$ and hence an attracting fixpoint of $f$.

Case (ii): Let $k=2$. It follows from (15) that $l=1$ and $g\left(z_{0}\right)-g\left(z_{0}\right)^{2} h\left(z_{0}\right)=1$ and hence $c a^{2}-a+1=0$ if $a=g\left(z_{0}\right)$ and $c=h\left(z_{0}\right)$. We have $f^{\prime}\left(z_{0}\right)=1-a$. If $|1-a|<1$, then $z_{0}$ is an attracting fixpoint of $f$. It is easy to verify that $|1-a|<1$ is equivalent to $\operatorname{Re}(1 / a)>\frac{1}{2}$. If $1-a$ is a root of unity, then $z_{0}$ is a rationally indifferent fixpoint of $f$. In the other cases, $z_{0}$ is a repelling or irrationally indifferent fixpoint of $f$. It is clear that $f^{n}$ cannot converge to a repelling fixpoint in some open set. In general, it is not known whether it is possible that the iterates of a meromorphic function converge to an irrationally indifferent fixpoint in some component of the Fatou set. It is known, however, that such a component must be a wandering domain, and thus we deduce from Theorem B that this situation does not occur in our case. Hence there does not exist an open set where $f^{n}$ converges to $z_{0}$ if $|1-a| \geqslant 1$ and if $1-a$ is not a root of unity.

The last claim that $z_{0}$ is a zero of $u$ if and only if $1 / a \in \mathbb{N}$ is obvious from the Laurent series of $u^{\prime} / u$. 
Case (iii): Let $k \geqslant 3$. It follows from (15) that $k=2 l$ with $l \in \mathbb{N} \backslash\{1\}$. Thus $z_{0}$ is a multiple zero of $u / u^{\prime}$. Therefore $f^{\prime}\left(z_{0}\right)=1$ and $z_{0}$ is a rationally indifferent fixpoint of $f$. Moreover, $u$ cannot be meromorphic at $z_{0}$ and thus $z_{0}$ is not a zero of $u$. This completes the proof.

Lemma 3. Let $z_{0}$ be a simple zero of $u / u^{\prime}$ such that $u(z) / u^{\prime}(z)=$ $a\left(z-z_{0}\right)+O\left(\left(z-z_{0}\right)^{2}\right)$ as $z \rightarrow z_{0}$. Then $u\left(z_{0}+r \mathrm{e}^{\mathrm{i} \theta}\right) \rightarrow 0$ as $r \rightarrow 0$ for any fixed branch of $u$ and $\theta \in \mathbb{R}$ if and only if $\operatorname{Re} a>0$.

Lemma 3 says that, even if $u$ is not meromorphic in a neighbourhood of $z_{0}$, we can still consider $z_{0}$ as a 'zero' of $u$ if $\operatorname{Re} a>0$. However, if $|1-a|>1$, there does not exist an open subset of $\mathbb{C}$ where $f^{n} \rightarrow z_{0}$ as $n \rightarrow \infty$. Hence values for which $\operatorname{Re} a>0$ and $|1-a|>1$ can be considered as 'zeros' of $u$ with no domain of attraction (see also Example 3 in §9). On the other hand, if $f^{n} \rightarrow z_{0}$ in some open set for a simple zero $z_{0}$ of $u / u^{\prime}$, then $z_{0}$ is a 'zero' of $u$ in the above sense.

There is no analogue of this for multiple zeros of $u / u^{\prime}$. For example, if $u(z)=$ $z^{2} \exp (1 / z)$, then $u$ satisfies (3) with $R(z)=-\left(2 z^{2}-2 z+1\right) / z^{4}, u(z) / u^{\prime}(z)=z^{2} /(2 z-1)$, and $f(z)=z(z-1) /(2 z-1)$. Thus $f^{n} \rightarrow 0$ in some open set as $n \rightarrow \infty$, but it does not seem to make sense to consider $z_{0}=0$ as a 'zero' of $u$.

Proof of Lemma 3. Let $u, z_{0}$ and $a$ be as in Lemma 3. Then $\log u(z)=$ $\log \left(z-z_{0}\right) / a+O(1)$, and hence $\log |u(z)|=\operatorname{Re}(1 / a) \log \left|z-z_{0}\right|-\operatorname{Im}(1 / a) \arg \left(z-z_{0}\right)$ $+O(1)$ as $z \rightarrow z_{0}$. Thus $\left|u\left(z_{0}+r \mathrm{e}^{\mathrm{i} \theta}\right)\right|=\exp \{\operatorname{Re}(1 / a) \log r-\operatorname{Im}(1 / a) \theta+O(1)\}$ as $r \rightarrow 0$. From this it is obvious that $\left|u\left(z_{0}+r \mathrm{e}^{\mathrm{i} \theta}\right)\right| \rightarrow 0$ for $r \rightarrow 0$ if and only if $\operatorname{Re}(1 / a)>0$ or, equivalently, if $\operatorname{Re} a>0$.

\section{Examples}

EXAmple 1. The simplest case occurs when $R$ is constant, say $R \equiv 1$. The general solution of (3) is given by $u(z)=a \sin z+b \cos z$. Theorem 1 says that Newton's method converges to zeros of $u$ on an open dense subset of $\mathbb{C}$, except when $a= \pm \mathrm{i} b$, in which case $u$ is of the form $u(z)=b \exp ( \pm \mathrm{i} z)$ excluded in Theorem 1 .

ExAmple 2. For $R(z)=-1 / z$, differential equation (3) has the entire transcendental solution

$$
u(z)=\sum_{n=1}^{\infty} \frac{1}{n !(n-1) !} z^{n} .
$$

Again we find that Newton's method for $u$ converges on an open dense subset of $\mathbb{C}$ to zeros of $u$.

EXAMPLE 3. Let

$$
R(z)=1-\frac{4 v^{2}-1}{4 z^{2}}
$$

where $v \in \mathbb{C}, \operatorname{Re} v \geqslant 0$. Because the case $R \equiv 1$ has already been considered in Example 1 , we can assume that $v \neq \frac{1}{2}$. A solution of (3) is given by $u_{v}(z)=\sqrt{z} J_{v}(z)$, where $J_{v}$ denotes the Bessel function of the first kind. For the corresponding Newton function $f_{v}$ we find that

$$
f_{v}(z)=z-\frac{2 z J_{v}(z)}{J_{v}(z)-2 z J_{v}^{\prime}(z)} .
$$


In the terminology of Lemma 2(ii), we have $c=-v^{2}+\frac{1}{4}$ and $a=2 /(1+2 v)$. We see that 0 is an attracting fixpoint of $f_{v}$ of multiplier $1-a=(2 v-1) /(2 v+1)$ if $\operatorname{Re} v>0$, and a rationally indifferent fixpoint of $f_{v}$ if $\operatorname{Re} v=0$ and $1-a=(2 v-1) /(2 v+1)$ is a root of unity.

We deduce that in these cases there is a critical point of $f_{v}$ and hence a zero of $R$ tending to 0 under iteration of $f_{v}$. Since $f_{v}$ is odd, we conclude that both zeros of $R$ have this property. Thus Theorem 1 implies that $f_{v}^{n}$ converges to 0 or to zeros of $J_{v}$ on an open dense subset of $\mathbb{C}$ if $\operatorname{Re} v>0$ or if $(2 v-1) /(2 v+1)$ is a root of unity, for example if $v=0$.

If $v$ is not an integer, then $u_{-v}(z)=\sqrt{z} J_{-v}(z)$ is a solution of (3), which is linearly independent of $u_{v}$. We now have $a=2 /(1-2 v)$ and deduce that, if $\operatorname{Re} v>0$, then 0 is a repelling fixpoint of

$$
f_{-v}(z)=z-\frac{2 z J_{-v}(z)}{J_{-v}(z)-2 z J_{-v}^{\prime}(z)}
$$

so that there are no open sets where $f_{-v}^{n}$ converges to 0 . Note that $\left|u_{-v}\left(r \mathrm{e}^{\mathrm{i} \theta}\right)\right| \rightarrow 0$ as $r \rightarrow 0$ if $\operatorname{Re} v<\frac{1}{2}$. This follows immediately from the definition of $J_{v}$, and also from Lemma 3.

The functions $u_{ \pm v}$ are meromorphic if and only if $v=m+\frac{1}{2}$ for some $m \in \mathbb{N}$. In this case, $u_{v}$ has a zero at 0 and $u_{-v}$ has a pole at 0 . In fact, for these values of $v$, the functions $u_{ \pm v}$ can be expressed in terms of trigonometric functions. For example, we have

$$
u_{3 / 2}(z)=\frac{\overline{2}}{\pi}\left(\frac{\sin z}{z}-\cos z\right)
$$

and

$$
u_{-3 / 2}(z)=\frac{\overline{2}}{\pi}\left(-\sin z-\frac{\cos z}{z}\right)
$$

The zeros of $u_{3 / 2}$ are the fixpoints of $\tan z$. As noted above, $f_{3 / 2}^{n}$ converges to zeros of $u_{3 / 2}$ on an open dense subset of $\mathbb{C}$.

The zeros of $u_{-3 / 2}$ are the solutions of

$$
z \tan z=-1
$$

For $v=\frac{3}{2}$, the zeros of $R$ are given by $\pm \sqrt{2}$. A numerical computation shows that $f_{-3 / 2}^{n}(\sqrt{2}) \rightarrow 2.798386 \ldots$ as $n \rightarrow \infty$. Because $f_{-3 / 2}$ is odd, we have $f_{-3 / 2}^{n}(-\sqrt{2}) \rightarrow$ $-2.798386 \ldots$ as $n \rightarrow \infty$. We deduce from Theorem 1 that $f_{-3 / 2}^{n}$ converges to solutions of (16) on an open dense subset of $\mathbb{C}$.

We remark that the solutions of (16) were computed in [29, p. 332] using Newton's method for the function $-1-z \tan z$ which leads to iteration of $\phi(z)=$ $\left(z^{2}-\cos z\right) /(z+\sin z \cos z)$. It is shown in [29] that, apart from the attracting fixpoints of $\phi$ corresponding to the solutions of (16), there are no attracting cycles for $\phi$. It is not clear (to us) whether $\phi$ has Baker domains or wandering domains.

In [29], the more general equation $z \tan z=c \in \mathbb{C}$ was also considered by applying Newton's method to $c-z \tan z$. Here, attractive cycles of higher order do occur for certain $c \neq-1$. The last equation was also studied in [21, 44 by the application of Newton's method to $z \sin z-c \cos z$. 
We now give the example mentioned in the introduction where $u$ is not meromorphic, $u / u^{\prime}$ is rational (of degree greater than 1), and Newton's method does not converge to zeros of $u$ or poles of $R$ on any open set, although $f^{n}\left(z_{j}\right)$ converges for all zeros of $R$.

EXAMPLE 4. Let

$$
R(z)=\frac{(7-\mathrm{i})(z-\mathrm{i})(z+1)}{z^{2}(5 z+3-4 \mathrm{i})^{2}} .
$$

Then

$$
u(z)=\exp \left(\frac{2+\mathrm{i}}{5} \log z+\frac{1}{5} \log \left(z+\frac{3-4 \mathrm{i}}{5}\right)\right)
$$

is a solution of (3). We obtain

$$
f(z)=-\frac{z(z+1-\mathrm{i})}{(1+\mathrm{i}) z+1} .
$$

The zeros of $R$ are i and -1 , and these are also the critical points of $f$. We have

$$
\mathrm{i} \stackrel{f}{\longrightarrow}-1 \stackrel{f}{\longrightarrow} 1 \stackrel{f}{\longrightarrow}-\frac{3}{5}+\frac{4}{5} \mathrm{i} .
$$

Moreover, $-\frac{3}{5}+\frac{4}{5} \mathrm{i}$ is a (repelling) fixpoint of $f$ so that $f^{n}\left(z_{j}\right)$ converges to a finite limit for all zeros of $R$. On the other hand, $F(f)=\varnothing$ since all critical points of $f$ are preperiodic but not periodic (compare, for example, [38, §5.1, Theorem 1]).

We mention that $f$ is conjugate to $g(z)=(z-2)^{2} / z^{2}$, and $g$ is a well known example of a function with empty Fatou set (see also [7, §11.9] for an elementary proof that $F(g)=\varnothing)$. We have taken $f$ instead of $g$ because the function $R$ corresponding to $g$ has multiple poles but does not satisfy (4).

Next we give two examples where the hypotheses of Theorem 1 are not satisfied and where, in fact, there are open sets where $f^{n}$ does not converge to zeros of $u$ or finite poles of $R$.

EXAmple 5. We consider the case $v=\frac{3}{2}$ of Example 3 in more detail. Then $R(z)=1-2 / z^{2}$. As noted above, the general soltion of (3) is meromorphic and given by

$$
u(z)=a\left(-\sin z-\frac{\cos z}{z}\right)+b\left(\frac{\sin z}{z}-\cos z\right) .
$$

As the case in which $a=0$ has already been studied in Example 3, we now assume that $a \neq 0$. The zeros of $u$ are then the solutions of $\tan z=(1+c z) /(c-z)$ where $c=$ $b / a$. The Newton function corresponding to $u$ is given by

$$
f_{c}(z)=z \frac{2+2 z \tan z-z^{2}+c\left(-2 \tan z+2 z+z^{2} \tan z\right)}{1+z \tan z-z^{2}+c\left(-\tan z+z+z^{2} \tan z\right)} .
$$

For $c \neq 0$, we also have the representation

$$
f_{c}(z)=2 z+\frac{z^{3}}{1-z^{2}+\frac{z}{c}\left(\frac{c^{2}+1}{1-c \tan (z)}-1\right)} .
$$


The zeros of $R$ are $\pm \sqrt{2}$ and we shall study their orbits under iteration of $f_{c}$. Because $f_{c}(-z)=-f_{-c}(z)$ and hence $f_{c}^{n}(-z)=-f_{-c}^{n}(z)$, it suffices to consider the orbit of $\sqrt{2}$. We have

$$
f_{c}(\sqrt{2})=\frac{4 \tan \sqrt{2}+4 c}{\sqrt{2} \tan \sqrt{2}-1+(\sqrt{2}+\tan \sqrt{2}) c}
$$

and we find that, if

$$
c=\frac{1+\sqrt{2} \tan \sqrt{2}}{\tan \sqrt{2}-\sqrt{2}}=2.023981360 \ldots,
$$

then $f_{c}(\sqrt{2})=\sqrt{2}$ so that $f_{c}$ has the superattracting fixpoint $\sqrt{2}$. For

$$
c=\frac{1-\sqrt{2} \tan \sqrt{2}}{\sqrt{2}+\tan \sqrt{2}}=-1.027033492 \ldots
$$

we have $f_{c}(\sqrt{2})=\infty$. The proof of Theorem 1 shows that the conclusion of Theorem 1 still holds for this value of $c$. On the other hand, it is fairly obvious that the behaviour for $c=-1.027033492 \ldots$ is unstable in the sense that slight perturbation of $c$ may yield different dynamics. In fact, the equation

$$
f_{c}^{2}(\sqrt{2})=\sqrt{2}
$$

may be written as $\tan \left(f_{c}(\sqrt{2})\right)=Q(c)$ with a rational function $Q$, and we can deduce from this that there are infinitely many solutions of (17) having $c=-1.027033492 \ldots$ as a limit point. Hence there are infinitely many values of $c$, accumulating at $-1.027033492 \ldots$, for which $f_{c}$ has a superattracting periodic point of period 2 . Numerical examples are $c=-0.335056289 \ldots$ with the superattracting cycle $\{\sqrt{2}, 4.47552177 \ldots\}, c=-1.793311697 \ldots$ with $\{\sqrt{2}, 11.4685457 \ldots\}$, and $c=$ $-17.76710140 \ldots$ with $\{\sqrt{2}, 0.352577052 \ldots\}$. Clearly, for these values of $c$, Newton's method does not converge to zeros of $u$ in the corresponding superattracting components.

Similarly, we can find superattracting periodic points of higher period. Here we only mention that $c=-9.486442848 \ldots$ leads to a superattracting periodic point of period 3.

In Figures 1-3, black indicates that the sequence $f_{c}^{n}(\sqrt{2})$ was not found to converge, white indicates that it converges but that many iterations were needed, and the various shades of grey indicate that convergence was observed after comparatively few iterations.

Figure 1 shows the set $-22<\operatorname{Re} c<2,|\operatorname{Im} z|<12$, and Figure 2 shows the set $-2.05<\operatorname{Re} c<0.25, \quad|\operatorname{Im} z|<1.15$. The complicated behaviour near $c=-1.027033492 \ldots$ is clearly visible.

Figure 3 shows the range $-0.3375<\operatorname{Re} c<-0.333,|\operatorname{Im} z|<0.00225$, containing the value $c=-0.3350568629 \ldots$ for which $f_{c}$ has a superattracting periodic point of period 2. The similarity to the Mandelbrot set is not surprising in view of the theory of polynomial-like mappings created by Douady and Hubbard [15]. In fact, there seem to be Mandelbrot-like sets around all values of $c$ for which $f_{c}$ has a superattracting periodic point. The one near $c=-17.76710140 \ldots$ is visible in Figure 1. 


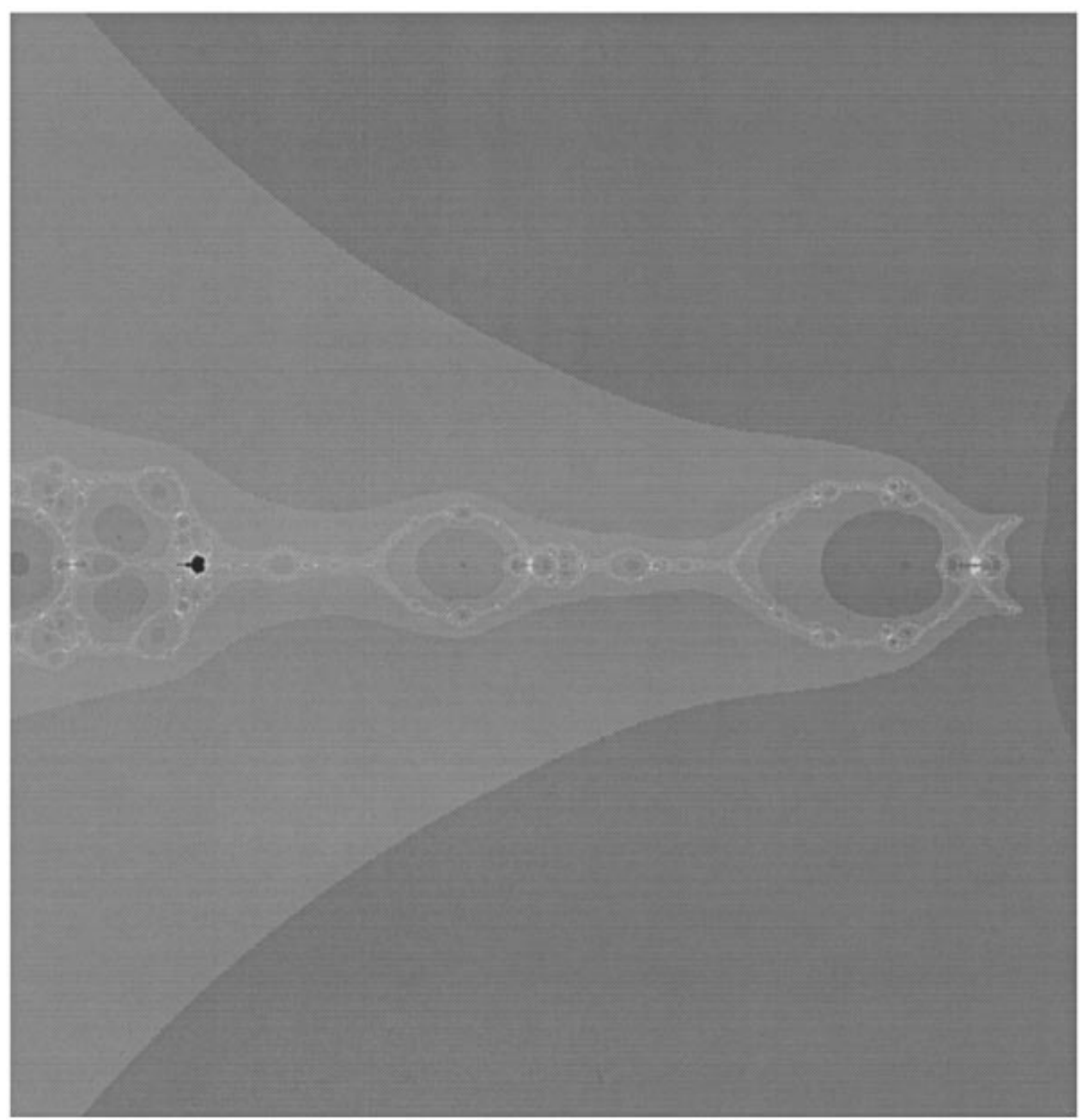

FIG. 1

Finally we consider $c= \pm$ i. We have

$$
f_{ \pm \mathrm{i}}(z)=2 z+\frac{z^{3}}{1-z^{2} \pm \mathrm{i} z}=z \frac{z^{2}-2 \mathrm{i} z-2}{z^{2} \mp \mathrm{i} z-1} .
$$

Hence $\infty$ is a rationally indifferent fixpoint of $f_{ \pm \mathrm{i}}$, and thus $f_{ \pm \mathrm{i}}^{n}$ tends to $\infty$ in the correponding parabolic domains. This implies that there is a zero of $R$ which tends to $\infty$ under iteration of $f_{ \pm \mathrm{i}}$. Because $f_{ \pm \mathrm{i}}(-\bar{z})=-\overline{f_{ \pm \mathrm{i}}(z)}$, we deduce that both zeros of $R$ have this property. Since the corresponding solutions of (3) are

$$
\frac{\mp \mathrm{i} a(z \mp \mathrm{i})}{z} \mathrm{e}^{\mp \mathrm{i} z}
$$

we conclude that, if $z \in F\left(f_{ \pm \mathrm{i}}\right)$, then $f_{ \pm \mathrm{i}}^{n}(z) \rightarrow \infty$ or $f_{ \pm \mathrm{i}}^{n}(z) \rightarrow \pm \mathrm{i}$ as $n \rightarrow \infty$.

In conclusion, we note that for many values of $c$ there are open sets where $f_{c}^{n}$ does not converge to zeros of $u$. 


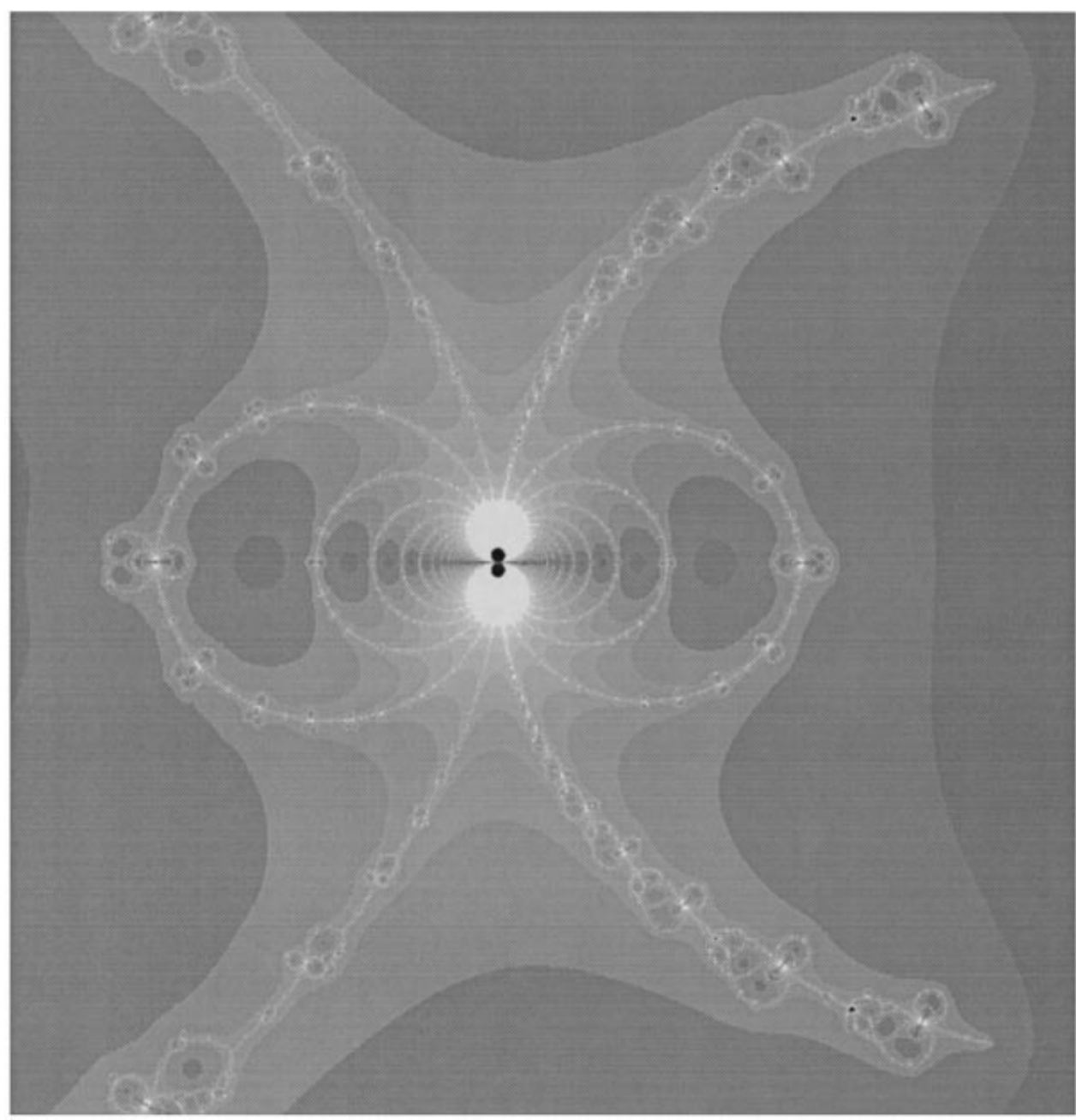

FIG. 2

Example 6. The Airy function $u(z)=\operatorname{Ai}(z)$ solves (3) for $R(z)=-z$. We have $\rho(u)=\frac{3}{2}$, and from the asymptotic expansions $[1$, p. 448] of $\operatorname{Ai}(z)$ we see that $\delta(0, u)$ $=\frac{1}{2}$. As in the proof of Theorem 2, we see that, if $\eta>0$ and $R=R(\eta)$ is sufficiently large, then $\{z:|z|>R,|\arg z|<\pi / 3-\eta\}$ is contained in an invariant Baker domain $U$ of $f$. Thus $f^{n}(z)$ tends to $\infty$ and not to zeros of $u$ for all $z \in U$, although $u$ is meromorphic.

From Theorems 1 and 2 and their proofs, we conclude that $0 \in U$ and hence $\left|f^{n}(0)\right| \rightarrow \infty$ as $n \rightarrow \infty$. In fact, from the remark at the end of $\S 7$, we deduce that

$$
\lim _{n \rightarrow \infty} \frac{\left|f^{n}(0)\right|^{3 / 2}}{n}=\frac{3}{2}
$$

We mention that the methods of $\S 2$ and $\S 7$ yield that, if $R$ is a non-constant polynomial, then (3) always has a solution such that the corresponding Newton function $f$ has an invariant Baker domain. 


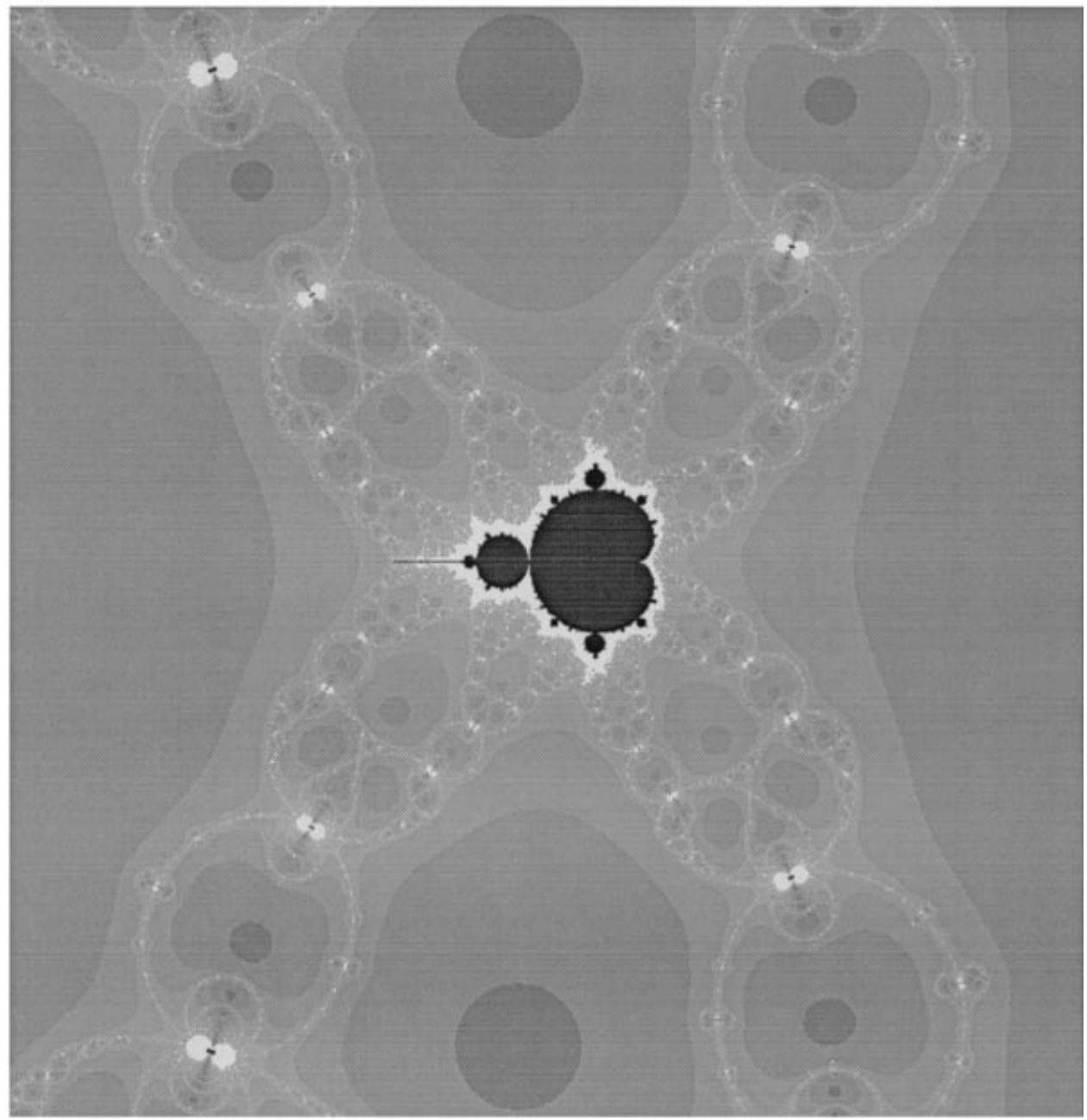

FIG. 3

Example 7. In [8, Theorem 3], a result similar to Theorem 1 was proved for Newton's method applied to

$$
w(z)=\int_{0}^{z} a(t) \mathrm{e}^{b(t)} d t+c
$$

where $a$ and $b$ are polynomials and $c$ is a constant. We note that $w$ satisfies (1) with $p=-a^{\prime} / a-b^{\prime}$ and $q=0$. We obtain (3) with

$$
u(z)=w(z) \frac{\mathrm{e}^{-b(z) / 2}}{\sqrt{a(z)}}
$$

and

$$
R(z)=-\frac{3}{4}\left(\frac{a^{\prime}}{a}\right)^{2}+\frac{1}{2} \frac{a^{\prime \prime}}{a}-\frac{1}{2} \frac{a^{\prime} b^{\prime}}{a}-\frac{1}{4} b^{\prime 2}+\frac{1}{2} b^{\prime \prime} .
$$

The application of Newton's method to $u$ instead of $w$, that is, the definition of $f$ by (6) instead of (5), has the advantage that the convergence is of the third order for all 
zeros of $w$ which are not zeros of $a$. On the other hand, the 'testpoints' in [8] are the zeros of $w^{\prime \prime}$ which are just the zeros of $a^{\prime}+a b^{\prime}$, while the 'testpoints' in Theorem 1 are the zeros of $R$. In general, $R$ will have more zeros than $a^{\prime}+a b^{\prime}$, so that, in some sense, (6) is more likely to lead to attracting cycles of higher period than (5). For the example

$$
w(z)=\int_{0}^{z} \mathrm{e}^{-t^{2}} d t
$$

considered in [8], we find that $p(z)=2 z, a^{\prime}(z)+a(z) b^{\prime}(z)=-a(z) p(z)=-2 z$ while $R(z)=-z^{2}-1$. Obviously 0 is a simple zero of $w$ and thus a superattracting fixpoint of the function $f$ defined by (5). By the results in [8], the iterates of the function $f$ defined by (5) converge to zeros of $w$ on an open dense subset of $\mathbb{C}$. If we define $f$ by (6), then we find from a numerical computation that $f^{n}( \pm \mathrm{i}) \rightarrow 0$ as $n \rightarrow \infty$. Since $\pm \mathrm{i}$ are the zeros of $R$, we deduce from Theorem 1 that the iterates of the function $f$ defined by (6) also converge to zeros of $w$ on an open dense subset of $\mathbb{C}$, the convergence being faster than for the function defined by (5).

Acknowledgements. We would like to thank Marcus Jankowski and Jim Langley for valuable comments, and the referee of this paper for a number of helpful suggestions that led to an improvement of the paper.

\section{References}

1. M. Abramowitz and I. A. Stegun, Handbook of mathematical functions (Dover, New York, 1965).

2. I. N. BAKER, 'Wandering domains in the iteration of entire functions', Proc. London Math. Soc. (3) 49 (1984) 563-576.

3. I. N. BAKER, J. KotUS and Y. Lü, 'Iterates of meromorphic functions I', Ergodic Theory Dynam. Systems 11 (1991) 241-248.

4. I. N. BAKER, J. KotUS and Y. LÜ, 'Iterates of meromorphic functions III: preperiodic domains', Ergodic Theory Dynam. Systems 11 (1991) 603-618.

5. I. N. BAKER, J. KotUS and Y. LÜ, 'Iterates of meromorphic functions IV: critically finite functions', Results Math. 22 (1992) 651-656.

6. S. BAnK and I. Laine, 'On the oscillation theory of $f^{\prime \prime}+A f=0$ where $A$ is entire', Trans. Amer. Math. Soc. 273 (1982) 351-363.

7. A. F. BeARDon, Iteration of rational functions (Springer, New York, 1991).

8. W. BERGWEILER, 'Newton's method and a class of meromorphic functions without wandering domains', Ergodic Theory Dynam. Systems 13 (1993) 231-247.

9. W. Bergweiler, 'Iteration of meromorphic functions', Bull. Amer. Math. Soc. 29 (1993) 151-188.

10. W. Bergweiler, 'Invariant domains and singularities', Math. Proc. Cambridge Philos. Soc. 117 (1995) $525-532$.

11. W. Bergweiler and N. Terglane, 'Weakly repelling fixpoints and the connectivity of wandering domains', Trans. Amer. Math. Soc. 348 (1996) 1-12.

12. W. Bergweiler, M. Haruta, H. Kriete, H.-G. Meier and N. Terglane, 'On the limit functions of iterates in wandering domains', Ann. Acad. Sci. Fenn. Ser. A I Math. 18 (1993) 369-375.

13. W. Bergweiler, F. von Haeseler, H. Kriete, H.-G. Meier and N. Terglane, 'Newton's method for meromorphic functions', Complex analysis and its applications, Pitman Research Notes in Mathematics 305 (eds. C. C. Yang, G. C. Wen, K. Y. Li and Y. M. Chiang; Pitman, 1994) $147-158$.

14. L. Carleson and T. W. Gamelin, Complex dynamics (Springer, New York, 1993).

15. A. Douady and J. H. Hubbard, 'On the dynamics of polynomial-like mappings', Ann. Sci. École Norm. Sup. (4) 18 (1985) 287-343.

16. A. E. Eremenko and M. Y. LyUbich, 'Iterates of entire functions', Dokl. Akad. Nauk. SSSR 279 (1984) (in Russian); Soviet Math. Dokl. 30 (1984) 592-594 (in English).

17. A. E. Eremenko and M. Y. Lyubich, 'Examples of entire functions with pathological dynamics', $J$. London Math. Soc. (2) 36 (1987) 458-468.

18. A. E. ERemenko and M. Y. LyUbich, 'Dynamical properties of some classes of entire functions', Ann. Inst. Fourier (Grenoble) 42 (1992) 989-1020.

19. P. Fatou, 'Sur les équations fonctionelles', Bull. Soc. Math. France 47 (1919) 161-271 and Bull. Soc. Math. France 48 (1920) 33-94, 208-314. 
20. P. Fatou, 'Sur l'itération des fonctions transcendantes entières', Acta Math. 47 (1926) 337-360.

21. S. Gelinas and R. Vaillancourt, 'Application of Julia-Fatou iteration theory in dielectric spectroscopy', Computational solution of nonlinear systems of equations, Lectures in Applied Mathematics 26 (eds. E. L. Allgower and F. George; American Mathematical Society, Providence, RI, 1990) 189-208.

22. L. R. Goldberg and L. Keen, 'A finiteness theorem for a dynamical class of entire functions', Ergodic Theory Dynam. Systems 6 (1986) 183-192.

23. G. Gundersen and E. Steinbart, 'A generalization of the Airy integral for $f^{\prime \prime}-z^{n} f=0$ ', Trans. Amer. Math. Soc. 337 (1993) 737-755.

24. W. K. Hayman, Meromorphic functions (Clarendon Press, Oxford, 1964).

25. M. Herman, 'Are there critical points on the boundaries of singular domains?', Comm. Math. Phys. 99 (1985) 593-612.

26. E. Hille, 'Zero point problems for linear differential equations of the second order', Mat. Tidsskrift $B$ (2) (1927) 25-44.

27. E. Hille, Ordinary differential equations in the complex domain (John Wiley, 1976).

28. A. HinkKanen, 'Iteration and the zeros of the second derivative of a meromorphic function', Proc. London Math. Soc. (3) 65 (1992) 629-650.

29. J. L. Howland and R. Vaillancourt, 'Attractive cycles in the iteration of meromorphic functions', Numer. Math. 46 (1985) 323-337.

30. A. Hurwitz and R. CourAnt, Vorlesungen über allgemeine Funktionentheorie und elliptische Funktionen (Springer, 4th ed., 1964).

31. G. JANK and L. Volkmann, Einführung in die Theorie der ganzen und meromorphen Funktionen mit Anwendungen auf Differentialgleichungen (Birkhäuser, 1985).

32. G. Julia, 'Sur l'itération des fonctions rationelles', J. Math. Pures Appl. 4 (1918) 47-245.

33. I. LaINE, Nevanlinna theory and complex differential equations (de Gruyter, New York, 1993).

34. J. Milnor, 'Dynamics in one complex variable: introductory lectures', Preprint 1990/5, Stony Brook Institute for Mathematical Sciences, 1990.

35. R. Nevanlinna, 'Über Riemannsche Flächen mit endlich vielen Windungspunkten', Acta Math. 58 (1932) 295-373.

36. R. Nevanlinna, Analytic functions (Springer, 1970).

37. G. M. Stallard, 'A class of meromorphic functions with no wandering domains', Ann. Acad. Sci. Fenn. Ser. A I Math. 16 (1991) 211-226.

38. N. SteinmetZ, Rational iteration (de Gruyter, 1993).

39. D. Sullivan, 'Quasiconformal homeomorphisms and dynamics I: solution of the Fatou-Julia problem on wandering domains', Ann. of Math. 122 (1985) 401-418.

40. H. Wittich, 'Eindeutige Lösungen der Differentialgleichung $w^{\prime}=R(z, w)$ ', Math. Z. 74 (1960) $278-288$.

Fachbereich Mathematik

Sekr. MA 8-2

TU Berlin

Straße des 17 Juni 136

D-10623 Berlin

Germany

Present address:

Mathematisches Seminar

CAU Kiel

Ludewig-Meyn-Straße 4

D-24098 Kiel

Germany

E-mail: bergweiler@math.uni-kiel.de
Lehrstuhl II für Mathematik

RWTH Aachen

D-52056 Aachen

Germany

E-mail: terglan@math2.rwth-aachen.de 